\title{
Nonalcoholic Fatty Liver Disease Is Associated with Increased Atrial Fibrillation Risk in an Elderly Chinese Population: A Cross-Sectional Study
}

\author{
Yu Zhang, ${ }^{1,2}$ Peifei Li, ${ }^{1}$ Min Miao, ${ }^{3}$ Yi Liu, ${ }^{1}$ Yue Pan, ${ }^{1}$ Lu Xu, ${ }^{2}$ Zhongwei Zhu, ${ }^{4}$ \\ Chengfu Xu, ${ }^{5}$ and Lei $X u$ iD ${ }^{1}$ \\ ${ }^{1}$ Department of Gastroenterology, Ningbo First Hospital, No. 59 Liuting Street, Haishu District, Ningbo, Zhejiang Province, China \\ ${ }^{2}$ Ningbo University, College of Medicine, No. 818 Fenghua Road, Jiangbei District, Ningbo, Zhejiang Province, China \\ ${ }^{3}$ Department of Gastroenterology, The Affiliated Hospital of Ningbo University, School of Medicine, No. 247 Renmin Road, \\ Jiangbei District, Ningbo, Zhejiang Province, China \\ ${ }^{4}$ Department of Gastroenterology, Zhenhai Lianhua Hospital, No. 168 Northern Tianyi Road, Jiaochuan Street, \\ Zhenhai District, Ningbo, China \\ ${ }^{5}$ Department of Gastroenterology, The First Affiliated Hospital, College of Medicine, Zhejiang University, No. 79 Qingchun Road, \\ Shangcheng District, Hangzhou, Zhejiang Province, China
}

Correspondence should be addressed to Lei Xu; xulei22@163.com

Received 9 December 2017; Revised 14 May 2018; Accepted 21 May 2018; Published 19 July 2018

Academic Editor: Senthil Kumar Venugopal

Copyright (c) 2018 Yu Zhang et al. This is an open access article distributed under the Creative Commons Attribution License, which permits unrestricted use, distribution, and reproduction in any medium, provided the original work is properly cited.

\begin{abstract}
Background. Atrial fibrillation and nonalcoholic fatty liver disease are two pathological conditions that are highly prevalent worldwide and share multiple CVD risk factors. There are rare researches performed among elderly adults. Aims. We conducted this cross-sectional analysis of elderly adults ( $\geq 65$ years) to investigate the association between atrial fibrillation and nonalcoholic fatty liver disease. Methods. We analyzed clinical data of the elderly adults ( $\geq 65$ years) who had health examination in Zhenhai Lianhua Hospital, Ningbo, China, in 2014. Results. 522 of the 1688 participants were diagnosed with nonalcoholic fatty liver disease, and 39 participants were diagnosed as having atrial fibrillation. Nonalcoholic fatty liver disease was associated with risk factors for AF in the elderly Chinese population (OR 1.95, 95\% CI 1.03-3.69). Adjustments for age, gender, systolic blood pressure, fasting plasma glucose, $\gamma$-glutamyl transpeptidase, high-density lipoprotein, triglycerides, total cholesterol and albumin, nonalcoholic fatty liver disease, and prevalent atrial fibrillation remained statistically significant (OR 2.76, 95\% CI 1.32-5.77). Conclusions. Our results show that nonalcoholic fatty liver disease is associated with an increased risk of atrial fibrillation in an elderly Chinese population.
\end{abstract}

\section{Introduction}

Atrial fibrillation (AF) is a growing public health problem [1]. Because of the aging population and improvements in cardiovascular treatments, its prevalence is expected to increase substantially over the next few decades [2]. AF has been reported to be associated with high rates of hospitalization and death [3]. Along with older age, there are many independent risk factors for AF like obesity, hypertension, diabetes, ischemic heart disease, heart failure, and valvular heart disease [4].
Nonalcoholic fatty liver disease (NAFLD) is one of the most prevalent liver diseases in the world whose prevalence ranges from $6 \%$ to $35 \%$, with a median of $20 \%$ in the general population [5]. In recent years, an increasing body of evidence has indicated that NAFLD is linked to cardiovascular disease [6], myocardial abnormalities [7], left ventricular diastolic dysfunction [8], heart failure [9], aortic valve sclerosis, and so on [10].

NAFLD has also been observed to be significantly associated with AF in patients with type 2 diabetes $[11,12]$. Furthermore, a cohort study showed that NAFLD was associated with 
an increased risk of prevalent AF in a middle-aged population [13]. However, whether the association between NAFLD and AF also holds true in the elderly population remains uncertain. Therefore, we conducted this cross-sectional study to explore the association between NAFLD and AF in an elderly Chinese population.

\section{Materials and Methods}

2.1. Participants. We conducted a cross-sectional study of the elderly adults ( $\geq 65$ years old) who had undergone an annual physical examination at Zhenhai Lianhua Hospital, Ningbo, China, in 2014. 1688 participants (930 males and 758 females) with a median age of 72 (68-76) years were included in this analysis. This study excluded the following participants: (1) those with unknown alcohol intake or excessive alcohol intake; (2) those with unknown BMI or BMI $\leq 18.0 \mathrm{~kg} / \mathrm{m}^{2}$; (3) those with incomplete basic physical data; (3) those with missing liver ultrasonic diagnosis; (4) those with unknown causes of chronic liver disease. This study was approved by the Hospital Ethics Committee. All the participants were verbally informed and agreed to participate in the study. Written informed consent was not required for the observational nature of the study.

2.2. Clinical Characteristics and Laboratory Data. Clinical examinations, including anthropometric and laboratory measurements, were performed using standard methods [14]. Height and weight were measured with basic clothing and without shoes. Body mass index (BMI) was calculated by the weight in kilograms divided by the square of height in meters. Blood pressure was recorded in a sitting position using a sphygmomanometer. Fasting blood samples were drawn from an antecubital vein for testing fasting plasma glucose (FPG), triglycerides (TG), total cholesterol (TC), high-density lipoprotein (HDL), low-density lipoprotein (LDL), and alanine aminotransferase (ALT), aspartate aminotransferase (AST), $\gamma$-glutamyl transpeptidase (GGT), serum uric acid, and albumin. All values were measured using an Olympus AU640 autoanalyzer (Olympus, Kobe, Japan) and standard methods.

2.3. Diagnosis of NAFLD. We diagnosed nonalcoholic fatty liver disease based on evidence of fatty liver according to abdominal ultrasonography using a Toshiba Nemio 20 sonography machine with a 3.5-MHz probe (Toshiba, Tokyo, Japan) and after excluding other etiology [15]. Routine ultrasonography evaluation of four intra-abdominal organs (liver, gallbladder, pancreas, and spleen) was performed by well-trained operators who were blind to the laboratory and clinical data. Diagnostic criteria of fatty liver are as follows: (i) increased liver brightness; (ii) diffuse hyperechogenicity of the liver compared to the kidneys; (iii) deep attenuation of hepatic echo; (iv) intrahepatic vessel borders and diaphragm [16].

2.4. Statistical Analysis. We made statistical analyses using SPSS 18.0 software for Windows (IBM SPSS, NY).
Continuous variables were compared by Student's t-test or the Mann-Whitney $U$ test and shown as the mean \pm SD (standard deviation) or the median and interquartile range. We compared categorical variables using the chi-square test or Fisher's exact test. We applied logistic regression analysis to assess the independent association between FLD and the prevalence of AF after adjustment for potential confounders. We considered a 2 -tailed test and a $P$ value less than 0.05 statistically significant.

\subsection{Results}

2.5.1. Clinical Characteristics of the Included Patients. Of the 1688 elderly participants included in this study, 522 (30.9\%, 269 males and 253 females) met the diagnostic criteria for NAFLD. Of the entire sample, 39 participants (2.3\%, 30 males and 9 females) had persistent or permanent AF.

The clinical characteristics of patients with AF or without AF are shown in Table 1. Compared with the participants without AF, those with AF were older, were more likely to be male, and had significantly higher values of AST, GGT, and SUA. BMI, diastolic blood pressure (DBP), systolic blood pressure (SBP), ALT, TC, LDL, HDL, TG, albumin, and FPG did not significantly differ between the two groups. Notably, as also shown in Table 1, the participants with AF had a significantly greater prevalence of NAFLD than those without AF.

When the participants were stratified by NAFLD status (Table 2), we found that the group with NAFLD contained more males and was slightly younger than the group without NAFLD. The values for BMI, SBP, DBP, LDL, TG, AST, ALT, GGT, albumin, FPG, and SUA were higher among the participants with NAFLD; however, they had lower values of HDL. Importantly, as also shown in Table 2, there was a marked difference in the prevalence of AF among participants with or without NAFLD.

2.5.2. Risk Factor Analysis for AF. As seen in Table 3, in the logistic regression analysis, relevant covariables were chosen as potential confounding factors based on their significance in the univariate analyses. In model 1, after adjustment for age and gender, the association between AF and NAFLD remained statistically significant (OR, 2.24; 95\% CI 1.18-4.29). Further adjustment for SBP, FPG, GGT, HDL, and TG did not remarkably change the study group's status, as seen in model 2 (OR 2.72; 95\% CI 1.30-5.67). In addition, age and GGT were independent predictors of AF in this model. In model 3, we added TC, and albumin based on model 3. In that model, the association between NAFLD and AF was still statistically significant (OR 2.76; 95\% CI 1.32-5.77).

2.5.3. Association between Liver Enzymes and the Prevalence Rate of AF. To explore the relationship between serum liver enzymes and the prevalence rate of AF, all the participants were classified into groups according to the upper limit of each serum liver enzyme concentration. For AST and ALT, we defined the normal level group as lower than 40U/L and elevated level group as higher than 40U/L. For GGT, the 
TABLE 1: Clinical characteristics of study participants stratified by AF status.

\begin{tabular}{|c|c|c|c|c|c|}
\hline Characteristic & All $(n=1688)$ & Without AF $(\mathrm{n}=1649)$ & With AF $(n=39)$ & t value & $P$-value \\
\hline Gender (n) (male/female) & $930 / 758$ & $900 / 749$ & $30 / 9$ & $7.689^{\mathrm{a}}$ & 0.006 \\
\hline Age (years) & $72(68-76)$ & $72(68-76)$ & $76(71-79)$ & $3.091^{\mathrm{b}}$ & 0.002 \\
\hline Body mass index $\left(\mathrm{kg} / \mathrm{m}^{2}\right)$ & $23.8 \pm 2.9$ & $23.8 \pm 2.9$ & $24.4 \pm 3.5$ & 1.132 & 0.265 \\
\hline Systolic blood pressure (mmHg) & $135.3 \pm 17.3$ & $135.4 \pm 17.3$ & $131.4 \pm 18.5$ & -1.422 & 0.155 \\
\hline Diastolic blood pressure $(\mathrm{mmHg})$ & $74.6 \pm 10.2$ & $74.6 \pm 10.2$ & $76.8 \pm 10.0$ & 1.360 & 0.174 \\
\hline Total cholesterol (mmol/L) & $4.9 \pm 1.0$ & $4.9 \pm 1.0$ & $4.7 \pm 1.3$ & -1.068 & 0.292 \\
\hline High-density lipoprotein $(\mathrm{mmol} / \mathrm{L})$ & $1.7 \pm 0.4$ & $1.7 \pm 0.4$ & $1.7 \pm 0.4$ & 0.176 & 0.860 \\
\hline Low-density lipoprotein (mmol/L) & $2.6 \pm 0.8$ & $2.6 \pm 0.8$ & $2.4 \pm 1.0$ & -1.096 & 0.280 \\
\hline Triglycerides $(\mathrm{mmol} / \mathrm{L})$ & $1.15(0.85-1.62)$ & $1.15(0.86-1.62)$ & $1.05(0.72-1.56)$ & $1.164^{\mathrm{b}}$ & 0.224 \\
\hline Aspartate aminotransferase (U/L) & $23(20-27)$ & $23(20-27)$ & $26(21-36)$ & $2.144^{\mathrm{b}}$ & 0.032 \\
\hline Alanine aminotransferase (U/L) & $17(13-23)$ & $17(13-23)$ & $18(14-27)$ & $1.140^{\mathrm{b}}$ & 0.254 \\
\hline$\gamma$-glutamyl transpeptidase $(\mathrm{U} / \mathrm{L})$ & $21(16-31)$ & $21(16-31)$ & $35(23-78)$ & $4.752^{\mathrm{b}}$ & 0.001 \\
\hline Albumin $(\mathrm{g} / \mathrm{L})$ & $45.5 \pm 2.7$ & $45.5 \pm 2.7$ & $45.2 \pm 2.3$ & -0.670 & 0.503 \\
\hline Fasting plasma glucose $(\mathrm{mmol} / \mathrm{L})$ & $5.34(4.93-5.96)$ & $5.33(4.93-5.96)$ & $5.52(4.85-5.94)$ & $0.301^{\mathrm{b}}$ & 0.763 \\
\hline Serum uric acid $(\mu \mathrm{mol} / \mathrm{L})$ & $340 \pm 85.9$ & $339 \pm 85.2$ & $394 \pm 100.0$ & 3.932 & 0.001 \\
\hline NAFLD (\%) & 30.9 & 30.6 & 46.2 & $4.335^{\mathrm{a}}$ & 0.037 \\
\hline
\end{tabular}

Data are expressed as mean $\pm S D$, median (IOQ), or percentage; a indicates $\chi 2$ test; $b$ indicates Mann-Whitney U test. NAFLD, nonalcoholic fatty liver disease; $\mathrm{AF}$, atrial fibrillation.

TABLE 2: Clinical characteristics of study participants stratified by NAFLD status.

\begin{tabular}{|c|c|c|c|c|}
\hline Characteristic & Without NAFLD (n=1166) & With NAFLD $(n=522)$ & t value & $P$-value \\
\hline Gender (n) (male/female) & $661 / 505$ & $269 / 253$ & 3.876 & 0.049 \\
\hline Age (years) & $72(68-77)$ & $71(68-75)$ & $-3.091^{b}$ & 0.002 \\
\hline Body mass index $\left(\mathrm{kg} / \mathrm{m}^{2}\right)$ & $23.0 \pm 2.5$ & $25.8 \pm 2.8$ & 19.452 & 0.001 \\
\hline Systolic blood pressure (mmHg) & $134.2 \pm 17.4$ & $137.7 \pm 16.9$ & 3.853 & 0.001 \\
\hline Diastolic blood pressure $(\mathrm{mmHg})$ & $74.0 \pm 10.1$ & $76.1 \pm 10.3$ & 4.046 & 0.001 \\
\hline Total cholesterol (mmol/L) & $4.89 \pm 0.99$ & $4.96 \pm 1.00$ & 1.314 & 0.189 \\
\hline High-density lipoprotein (mmol/L) & $1.7 \pm 0.4$ & $1.5 \pm 0.3$ & -11.008 & 0.001 \\
\hline Low-density lipoprotein $(\mathrm{mmol} / \mathrm{L})$ & $2.6 \pm 0.8$ & $2.7 \pm 0.8$ & 1.542 & 0.123 \\
\hline Triglycerides $(\mathrm{mmol} / \mathrm{L})$ & $1.02(0.78-1.45)$ & $1.47(1.10-2.04)$ & $-13.114^{\mathrm{b}}$ & 0.001 \\
\hline Aspartate aminotransferase (U/L) & $23(20-27)$ & $24(20-30)$ & $-2.936^{\mathrm{b}}$ & 0.003 \\
\hline Alanine aminotransferase (U/L) & $16(12-21)$ & $20(15-28)$ & $-9.946^{\mathrm{b}}$ & 0.001 \\
\hline$\gamma$-glutamyl transpeptidase $(\mathrm{U} / \mathrm{L})$ & $20(16-29)$ & $26(19-37)$ & $-9.656^{\mathrm{b}}$ & 0.001 \\
\hline Albumin $(g / L)$ & $45.3 \pm 2.7$ & $45.8 \pm 2.7$ & 3.400 & 0.001 \\
\hline Fasting plasma glucose $(\mathrm{mmol} / \mathrm{L})$ & $5.26(4.86-5.82)$ & $5.52(5.12-6.27)$ & $-6.957^{\mathrm{b}}$ & 0.001 \\
\hline Serum uric acid (umol/L) & $329 \pm 81.6$ & $367 \pm 89.2$ & 8.722 & 0.001 \\
\hline $\mathrm{AF}(\%)$ & 1.8 & 3.4 & $4.335 \mathrm{a}$ & 0.037 \\
\hline
\end{tabular}

Data are expressed as mean \pm SD, median (IOQ), or percentage; a indicates $\chi 2$ test; $b$ indicates Mann-Whitney U test. NAFLD, nonalcoholic fatty liver disease; AF, atrial fibrillation.

upper limit was 50U/L for males and 32U/L for females. The prevalence rate of $\mathrm{AF}$ in the groups with different levels of AST, ALT, and GGT was analyzed.

As seen in Figure 1, there was an increasing trend of the prevalence rate of $\mathrm{AF}$ as serum liver enzyme increased. The prevalence rates of $\mathrm{AF}$ in normal and elevated serum AST groups are $2.0 \%$ and $9.6 \%$. And this trend is significant (Figure 1; $P<0.001$ ). Meanwhile, the rates in normal and elevated serum ALT/GGT groups are $2.1 \%$ and $7.8 \% / 1.6 \%$ and $7.2 \%$, with significant result as well (Figure 2; $P=$ $0.004 /<0.001)$. These results showed that participants with higher serum liver enzyme are more likely to develop AF than those with lower one.

Additionally, we combined the serum liver enzyme and NAFLD status to further investigate the relationship between NAFLD and AF (Figure 2). The participants were classified into three groups: without NAFLD, NAFLD with normal concentration of AST/ALT/GGT, and NAFLD with elevated AST/ALT/GGT concentration. The classification boundary value was as shown in Figure 2. The prevalence rates of AF among the different groups were analyzed. Figure 2 shows that those with hepatic steatosis, irrespective of serum liver 


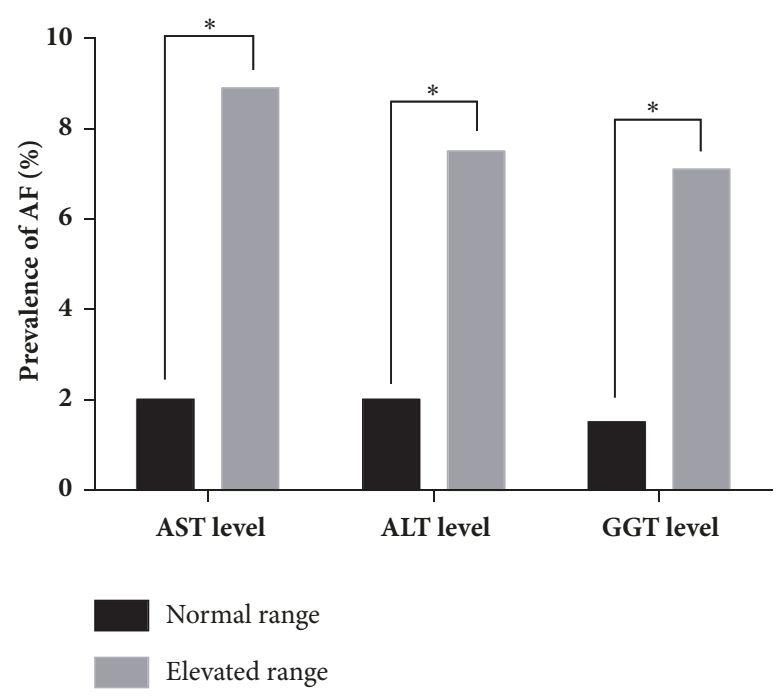

Figure 1: Prevalence of AF in an elderly Chinese population stratified by normal or elevated serum liver enzyme concentration. Normal range for AST or AST level: $\leq 40 \mathrm{U} / \mathrm{L}$; elevated range for AST or ALT level: $>40 \mathrm{U} / \mathrm{L}$; normal range for GGT level in male: $\leq 50 \mathrm{U} / \mathrm{L}$; elevated range for GGT in male: $>50 \mathrm{U} / \mathrm{L}$; normal range for GGT level in female: $\leq 32 \mathrm{U} / \mathrm{L}$; elevated range for GGT in female: $>32 \mathrm{U} / \mathrm{L}$. $* P$ value $<0.001 /=0.004 /<0.001$ by the $\chi 2$ test in AST/ALT/GGT group.

TABLE 3: Association between NAFLD and risk of prevalent AF in patients in an elderly population. Model 1: age and gender; model 2: age, gender, systolic blood pressure, fasting plasma glucose, $\gamma$ glutamyl transpeptidase, high-density lipoprotein, and triglycerides; model 3: model 2 plus total cholesterol and albumin.

\begin{tabular}{lcc}
\hline NAFLD (yes compared with no) & OR (95\% CI) & $P$-value \\
\hline Unadjusted model & $1.95(1.03-3.69)$ & 0.041 \\
Adjusted model 1 & $2.24(1.18-4.29)$ & 0.014 \\
Adjusted model 2 & $2.72(1.30-5.67)$ & 0.008 \\
Adjusted model 3 & $2.76(1.32-5.77)$ & 0.007 \\
\hline Other independent predictors of AF in model 2 & \\
Age & $1.11(1.05-1.18)$ & 0.001 \\
$\gamma$-glutamyl transpeptidase & $1.01(1.01-1.02)$ & 0.001 \\
\hline
\end{tabular}

NAFLD, nonalcoholic fatty liver disease; AF, atrial fibrillation.

enzyme level, had the highest prevalence of AF. However, the presence of AF among those without hepatic steatosis on ultrasound was negligible.

\section{Discussion}

At present, NAFLD and AF are known to be two pathological conditions that are highly prevalent worldwide and share multiple CVD risk factors. In recent years, published studies about the association between AF and NAFLD (or liver transaminase concentrations) have increased [11-13, 17, 18]. The study by Targher et al. was limited to subjects with type 2 diabetes [11, 19], and the OPERA study focused on middle-aged participants [13]. What is more, after systematic analysis, several reviews claim similar conclusions and explain the mechanisms between two relative diseases [2023]. As research performed among elderly adults is rare, we conducted this cross-sectional analysis of elderly adults ( $\geq 65$ years) to investigate the association between NAFLD and AF.
The main finding of the present study was that NAFLD is associated with AF in an elderly Chinese population. The logistic regression also showed that NAFLD and prevalent AF were correlated (OR 1.95, 95\% CI 1.03-3.69). Furthermore, the serum transaminase concentration was significantly associated with AF.

It is unknown whether these two diseases just share common pathophysiologic mechanisms or the association between them is causative. As NAFLD is a known risk factor for a wide range of cardiovascular diseases, it is reasonable to deduce that there may be a causal link. The following could be part of the explanation for our findings.

First, liver transaminases may link NAFLD and AF. The Framingham Heart Study demonstrated an independent relationship in the general adult population between liver transaminase concentrations and the risk of new-onset AF [24]. Targher et al's research proposed that, in subjects with type 2 diabetes, GGT was the only liver enzyme that was significantly associated with the prevalence of AF [12]. A similar result was found in an elderly Chinese population. GGT is a systemic marker of NAFLD [17]. The GGT level cannot be independent of NAFLD, so the development of the two diseases may be parallel. In addition, in our study, AST and ALT showed the same trend as GGT with the prevalence of AF. Furthermore, it has been proven that ALT is independently associated with an increased risk of cardiovascular related mortality, and ALT is the most specific marker of liver pathology [25-27]. However, Wang et al. supposed that the inflammatory response may be weaker in the aged liver [28] because lower ALT has been associated with increased mortality in the elderly [29]. Like Wang et al's study, the present investigation reported no mortality data. However, we must pay attention to high levels of ALT, especially in patients with NAFLD. As AST is produced not only in the liver, but also in the myocardium, it increases both NAFLD and AF in patients with both conditions. This 


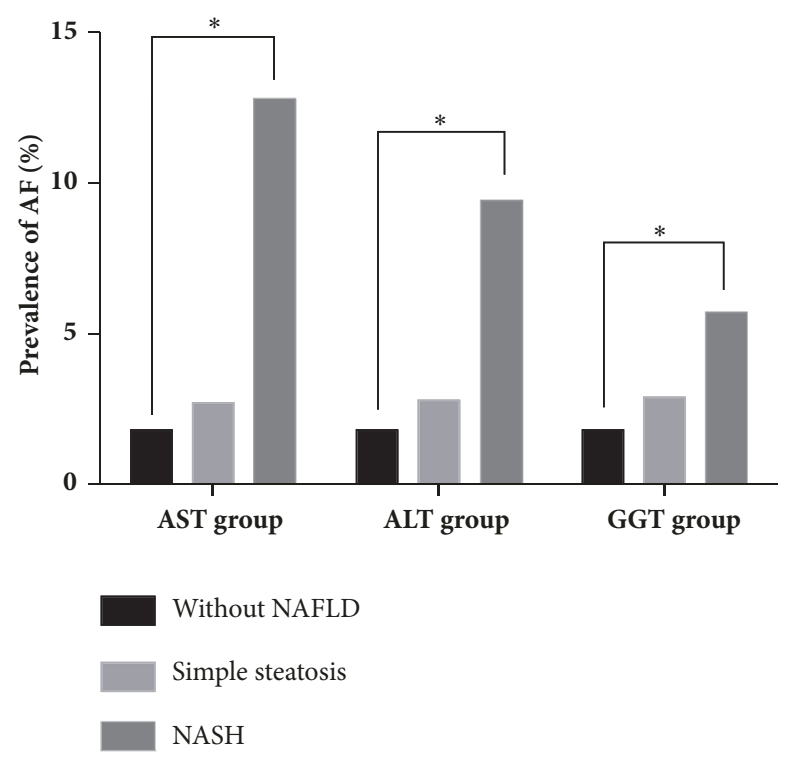

FIgURE 2: Prevalence of AF in an elderly Chinese population stratified by NAFLD status on ultrasound combined with normal or elevated serum AST/ALT/GGT concentration. Without NAFLD: without diagnosed NAFLD. Simple steatosis: NAFLD with normal range of AST/ALT/GGT. NASH: NAFLD with elevated range of AST/ALT/GGT. Normal range for AST or AST level: $\leq 40 \mathrm{U} / \mathrm{L}$; elevated range for AST or ALT level: > $40 \mathrm{U} / \mathrm{L}$; normal range for GGT level in male: $\leq 50 \mathrm{U} / \mathrm{L}$; elevated range for GGT in male: $>50 \mathrm{U} / \mathrm{L}$; normal range for GGT level in female: $\leq 32 \mathrm{U} / \mathrm{L}$; elevated range for GGT in female: $>32 \mathrm{U} / \mathrm{L} . * P$ value $=0.001 /=0.003 /=0.027$ by the $\chi 2$ test in AST/ALT/GGT group.

is consistent with our results. Therefore, good control of liver transaminase concentrations may help to reduce the mortality of AF.

Second, NAFLD provokes systemic inflammation to aggravate AF. The accumulation of impaired lipoprotein and hepatic lipid and increased oxidative stress in hepatic cells may induce oxidative stress and cause the secretion of inflammatory factors $[30,31]$. Previous studies have shown that chronic inflammation and oxidative stress are important risk factors for AF [32, 33]. Notably, many inflammatory factors produced by NAFLD [34] may cause AF. Furthermore, AF has been reported to be an trigger of an inflammatory environment [33], thus creating a vicious cycle. Remarkably, lipoprotein (HDL, LDL) can prevent the adhesion of bacteria and protest against endotoxemia or inflammation [35, 36]. The persistence of low levels of HDL and LDL could cause further lipoprotein consumption. These conclusions were consistent with our results shown in Table 1: HDL cholesterol, LDL cholesterol, and triglycerides were all lower in group with AF than in the group without AF.

Third, as NAFLD includes multiple cardiovascular risk factors, it may induce AF directly through several mechanisms. Some research has shown that NAFLD can cause left ventricular diastolic dysfunction [8,37], and other studies have indicated that NAFLD alters atrial conduction properties [7, 38]. Fat may accumulate in not only the liver tissue, but also other viscera and organs. When it settles in the pericardium or myocardium, the diastolic function of the ventricle or atria is harmed $[39,40]$. Moreover, increased fat can infiltrate the atrial septum and lead to electromechanical changes. It is important to provide health education to elderly patients with NAFLD to ensure that they develop healthier daily habits and get proper exercise.

Finally, many studies have reported that NAFLD is an independent risk factor for autonomic dysfunction [41-43]. Additionally, Sun et al. showed results that correspond to those of our study [43]. There are also other reports that showed that autonomic dysfunction is a risk factor for $\mathrm{AF}$ $[44,45]$. These results may partly explain the relationship between NAFLD and AF.

Though there have been many articles showing similar information as shown in this study, the unique part is as follows: Firstly, research performed among elderly adults is rare and the elderly population is increasing worldwide [46]. Since China became a aging country in 2000, the development trend of the aging population, the health of the elderly population, and related social problems have all been taken seriously. It is our responsibility to pay more attention to the health of the elderly. Secondly, the prevalence of NAFLD among Chinese and foreigners is different $[47,48]$. Since many international standards are not fully applicable to Chinese people, this study can provide health guidance for the larger Chinese population.

There are some limits to our study. First, though ultrasound-based diagnosis of NAFLD is widely used in clinic as a noninvasive and cost-effective method for hepatic steatosis screening, it cannot replace the gold standard, pathological study, for disease diagnosis. Second, with consideration of the prevalence of these diseases, the sample of our study is small. If we can get more support from the medical center, we will carry out multicenter or cohort research in the future to further confirm and improve our conclusions. At present, there are many formal researches for 
us to further validate the conclusions. Third, we diagnose $\mathrm{AF}$ according to resting electrocardiograms for absence of 24-hour dynamic electrocardiogram which is more precise but more difficult to accomplish during health examinations. Fourth, as our data was cross-sectional, we did not know the most recent mean levels of some laboratory results for each participant. As formal cohort studies have shown, NAFLD is associated with an increased risk of prevalent $\operatorname{AF}[12,13,49]$. What is more, Zhou YQ et al. [20] and Ding YH et al. [21] illustrated possible pathological mechanisms responsible for the association between NAFLD and increased risk of AF. Therefore, we can draw a conclusion regarding cause and effect.

In conclusion, NAFLD is associated with AF in an elderly Chinese population. In addition, based on previous studies, we can conclude that NAFLD is associated with an increased AF risk in an elderly Chinese population.

\section{Conflicts of Interest}

The authors declare that they have no conflicts of interest.

\section{Authors' Contributions}

Yu Zhang, Lei $\mathrm{Xu}$, and Chengfu $\mathrm{Xu}$ designed this study; Zhongwei Zhu, Min Miao, and $\mathrm{Lu} \mathrm{Xu}$ collected clinical data. Peifei Li, Yi Liu, and Yue Pan took part in statistical analysis. Yu Zhang drafted the manuscript and made the final approval of the version to be published.

\section{Acknowledgments}

This work was supported by National Natural Science Foundation of China (no. 81300703 to $\mathrm{Lu} \mathrm{Xu}$, no. 81470838 to Chengfu Xu), Zhejiang Provincial Natural Science Foundation of China (no. LY13H030012 to Lu Xu, no. LR15H030001 to Chengfu $\mathrm{Xu}$ ), and Ningbo Natural Science Foundation of China (no. 2015A610178 to Yue Pan, no. 2015A610183 to Yi Liu).

\section{References}

[1] G. Y. H. Lip, H. F. Tse, and D. A. Lane, "Atrial fibrillation," The Lancet, vol. 379, no. 9816, pp. 648-661, 2012.

[2] Miyasaka, "Erratum: Secular trends in incidence of atrial fibrillation in Olmsted County, Minnesota, 1980 to 2000, and implications on the projections for future prevalence (Circulation (2006) 114, (119-125))," Circulation, vol. 114, no. 11, p. e498, 2006.

[3] P. Jabre, V. L. Roger, M. H. Murad et al., "Mortality associated with atrial fibrillation in patients with myocardial infarction: a systematic review and meta-analysis," Circulation, vol. 123, no. 15, pp. 1587-1593, 2011.

[4] B. M. Psaty, T. A. Manolio, L. H. Kuller et al., "Incidence of and risk factors for atrial fibrillation in older adults," Circulation, vol. 96, no. 7, pp. 2455-2461, 1997.

[5] G. Vernon, A. Baranova, and Z. M. Younossi, "Systematic review: the epidemiology and natural history of non-alcoholic fatty liver disease and non-alcoholic steatohepatitis in adults,"
Alimentary Pharmacology \& Therapeutics, vol. 34, no. 3, pp. 274-285, 2011.

[6] G. Targher, C. P. Day, and E. Bonora, "Risk of cardiovascular disease in patients with nonalcoholic fatty liver disease," The New England Journal of Medicine, vol. 363, no. 14, pp. 1341-1350, 2010.

[7] A. Mantovani, S. Ballestri, A. Lonardo, and G. Targher, "Cardiovascular Disease and Myocardial Abnormalities in Nonalcoholic Fatty Liver Disease," Digestive Diseases and Sciences, vol. 61, no. 5, pp. 1246-1267, 2016.

[8] F. Fallo, A. Dalla Pozza, N. Sonino et al., "Non-alcoholic fatty liver disease is associated with left ventricular diastolic dysfunction in essential hypertension," Nutrition, Metabolism \& Cardiovascular Diseases, vol. 19, no. 9, pp. 646-653, 2009.

[9] F. Valbusa, S. Bonapace, C. Grillo et al., "Nonalcoholic fatty liver disease is associated with higher 1-year all-cause rehospitalization rates in patients admitted for acute heart failure," Medicine (United States), vol. 95, no. 7, p. e2760, 2016.

[10] S. Ballestri, A. Lonardo, S. Bonapace, C. D. Byrne, P. Loria, and G. Targher, "Risk of cardiovascular, cardiac and arrhythmic complications in patients with non-alcoholic fatty liver disease," World Journal of Gastroenterology, vol. 20, no. 7, pp. 1724-1745, 2014.

[11] G. Targher, A. Mantovani, I. Pichiri et al., "Non-alcoholic fatty liver disease is associated with an increased prevalence of atrial fibrillation in hospitalized patients with type 2 diabetes," Clinical Science, vol. 125, no. 6, pp. 301-309, 2013.

[12] G. Targher, F. Valbusa, S. Bonapace et al., "Non-alcoholic fatty liver disease is associated with an increased incidence of atrial fibrillation in patients with type 2 diabetes," PLOS ONE, vol. 8, no. 2, Article ID e57183, 2013.

[13] A. J. Käräjämäki, O.-P. Pätsi, M. Savolainen, Y. A. Kesäniemi, H. Huikuri, and O. Ukkola, "Non-alcoholic fatty liver disease as a predictor of atrial fibrillation in middle-aged population (OPERA study)," PLoS ONE, vol. 10, no. 11, Article ID e0142937, 2015.

[14] Y. Li, C. Xu, C. Yu, L. Xu, and M. Miao, "Association of serum uric acid level with non-alcoholic fatty liver disease: a crosssectional study," Journal of Hepatology, vol. 50, no. 5, pp. 10291034, 2009.

[15] C. Cerda, R. M. Pérez-Ayuso, A. Riquelme et al., "Nonalcoholic fatty liver disease in women with polycystic ovary syndrome," Journal of Hepatology, vol. 47, no. 3, pp. 412-417, 2007.

[16] R. Hernaez, M. Lazo, S. Bonekamp et al., "Diagnostic accuracy and reliability of ultrasonography for the detection of fatty liver: a meta-analysis," Hepatology, vol. 54, no. 3, pp. 1082-1090, 2011.

[17] M. R. P. Markus, P. J. Meffert, S. E. Baumeister et al., "Association between hepatic steatosis and serum liver enzyme levels with atrial fibrillation in the general population. The Study of Health in Pomerania (SHIP)," Atherosclerosis, vol. 245, pp. 123-131, 2016.

[18] G. A. Makar, M. G. Weiner, S. E. Kimmel et al., "Incidence and prevalence of abnormal liver associated enzymes in patients with atrial fibrillation in a routine clinical care population," Pharmacoepidemiology and Drug Safety, vol. 17, no. 1, pp. 43-51, 2008.

[19] G. Targher, L. Bertolini, F. Poli et al., "Nonalcoholic fatty liver disease and risk of future cardiovascular events among type 2 diabetic patients," Diabetes, vol. 54, no. 12, pp. 3541-3546, 2005.

[20] Y. Zhou, C. Lai, C. Peng et al., "Nonalcoholic fatty liver disease as a predictor of atrial fibrillation: a systematic review and 
meta-analysis," Advances in Interventional Cardiology/Postępy w Kardiologii Interwencyjnej, vol. 3, pp. 250-257, 2017.

[21] Y.-H. Ding, Y. Ma, L.-Y. Qian et al., "Linking atrial fibrillation with non-alcoholic fatty liver disease: Potential common therapeutic targets," Oncotarget, vol. 8, no. 36, pp. 60673-60683, 2017.

[22] K. Wijarnpreecha, B. Boonpheng, C. Thongprayoon, V. Jaruvongvanich, and P. Ungprasert, "The association between nonalcoholic fatty liver disease and atrial fibrillation: A metaanalysis," Clinics and Research in Hepatology and Gastroenterology, vol. 41, no. 5, pp. 525-532, 2017.

[23] A. M. Minhas, M. S. Usman, M. S. Khan, K. Fatima, M. A. Mangi, and M. S. Illovsky, "Link Between Non-Alcoholic Fatty Liver Disease and Atrial Fibrillation: A Systematic Review and Meta-Analysis," Cureus, vol. 9, p. E1142, 2017.

[24] M. F. Sinner, N. Wang, C. S. Fox et al., "Relation of circulating liver transaminase concentrations to risk of new-onset atrial fibrillation," American Journal of Cardiology, vol. 111, no. 2, pp. 219-224, 2013.

[25] J. P. Ong, A. Pitts, and Z. M. Younossi, "Increased overall mortality and liver-related mortality in non-alcoholic fatty liver disease," Journal of Hepatology, vol. 49, no. 4, pp. 608-612, 2008.

[26] C. Söderberg, P. Stål, J. Askling et al., "Decreased survival of subjects with elevated liver function tests during a 28 -year follow-up," Hepatology, vol. 51, no. 2, pp. 595-602, 2010.

[27] G. N. Ioannou, N. S. Weiss, E. J. Boyko, D. Mozaffarian, and S. P. Lee, "Elevated serum alanine aminotransferase activity and calculated risk of coronary heart disease in the United States," Hepatology, vol. 43, no. 5, pp. 1145-1151, 2006.

[28] Z. Wang, M. Xu, J. Peng et al., "Prevalence and associated metabolic factors of fatty liver disease in the elderly," Experimental Gerontology, vol. 48, no. 8, pp. 705-709, 2013.

[29] I. Ford, S. P. Mooijaart, S. Lloyd et al., "The inverse relationship between alanine aminotransferase in the normal range and adverse cardiovascular and non-cardiovascular outcomes," International Journal of Epidemiology, vol. 40, no. 6, Article ID dyr172, pp. 1530-1538, 2011.

[30] S. Furukawa, T. Fujita, M. Shimabukuro et al., "Increased oxidative stress in obesity and its impact on metabolic syndrome," The Journal of Clinical Investigation, vol. 114, no. 12, pp. 1752-1761, 2004.

[31] C. E. Ndumele, K. Nasir, R. D. Conceiçao, J. A. M. Carvalho, R. S. Blumenthal, and R. D. Santos, "Hepatic steatosis, obesity, and the metabolic syndrome are independently and additively associated with increased systemic inflammation," Arteriosclerosis, Thrombosis, and Vascular Biology, vol. 31, no. 8, pp. 1927-1932, 2011.

[32] M. Harada, D. R. Van Wagoner, and S. Nattel, "Role of inflammation in atrial fibrillation pathophysiology and management," Circulation Journal, vol. 79, no. 3, pp. 495-502, 2015.

[33] Y. Guo, G. Y. H. Lip, and S. Apostolakis, "Inflammation in atrial fibrillation," Journal of the American College of Cardiology, vol. 60, no. 22, pp. 2263-2270, 2012.

[34] G. Targher and C. Byrne, "Diagnosis and management of nonalcoholic fatty liver disease and its hemostatic/thrombotic and vascular complications," Seminars in Thrombosis and Hemostasis, vol. 39, no. 2, pp. 214-228, 2013.

[35] J. F. P. Berbée, L. M. Havekes, and P. C. N. Rensen, "Apolipoproteins modulate the inflammatory response to lipopolysaccharide," Journal of Endotoxin Research, vol. 11, no. 2, pp. 97-103, 2005.
[36] P. Patel, H. Dokainish, P. Tsai, and N. Lakkis, "Update on the Association of Inflammation and Atrial Fibrillation," Journal of Cardiovascular Electrophysiology, vol. 21, no. 9, pp. 1064-1070, 2010.

[37] L. J. Rijzewijk, R. W. van der Meer, J. W. A. Smit et al., "Myocardial steatosis is an independent predictor of diastolic dysfunction in type 2 diabetes mellitus," Journal of the American College of Cardiology, vol. 52, no. 22, pp. 1793-1799, 2008.

[38] O. Ozveren, C. Izgi, E. Eroglu et al., "Doppler tissue evaluation of atrial conduction properties in patients with non-alcoholic fatty-liver disease," Ultrasonic Imaging, vol. 38, no. 3, pp. 225235, 2015.

[39] C. S. Fox, P. Gona, U. Hoffmann et al., "Pericardial fat, intrathoracic fat, and measures of left ventricular structure and function," Circulation, vol. 119, no. 12, pp. 1586-1591, 2009.

[40] P.-S. Chen and I. Turker, "Epicardial adipose tissue and neural mechanisms of atrial fibrillation," Circulation: Arrhythmia and Electrophysiology, vol. 5, no. 4, pp. 618-620, 2012.

[41] J. L. Newton, J. Pairman, K. Wilton, D. E. J. Jones, and C. Day, "Fatigue and autonomic dysfunction in non-alcoholic fatty liver disease," Clinical Autonomic Research, vol. 19, no. 6, pp. 319-326, 2009.

[42] Y.-C. Liu, C.-S. Hung, Y.-W. Wu et al., "Influence of nonalcoholic fatty liver disease on autonomic changes evaluated by the time domain, frequency domain, and symbolic dynamics of heart rate variability," PLOS ONE, vol. 8, no. 4, article e61803, 2013.

[43] W. Sun, D. Zhang, and J. Sun, "Association between nonalcoholic fatty liver disease and autonomic dysfunction in a Chinese population," QJM: An International Journal of Medicine, vol. 108, no. 8, pp. 617-624, 2015.

[44] H.-W. Park, M. J. Shen, S.-F. Lin, M. C. Fishbein, L. S. Chen, and P.-S. Chen, "Neural mechanisms of atrial fibrillation," Current Opinion in Cardiology, vol. 27, no. 1, pp. 24-28, 2012.

[45] M. J. Shen and D. P. Zipes, "Role of the autonomic nervous system in modulating cardiac arrhythmias," Circulation Research, vol. 114, no. 6, pp. 1004-1021, 2014.

[46] G. Daradkeh, M. M. Essa, S. Samir Al-Adawi, R. P. Koshy, A. Al-Asmi, and M. I. Waly, "Nutritional status and cognitive impairment in elderly," Pakistan Journal of Biological Sciences, vol. 17, no. 10, pp. 1098-1105, 2014.

[47] J. Fan, S. Kim, and V. W. Wong, "New trends on obesity and NAFLD in Asia," Journal of Hepatology, vol. 67, no. 4, pp. 862873, 2017.

[48] Y. M. Nan, N. Fu, W. C. Li, and et al., "An interpretation of the AASLD practice guideline on the diagnosis and management of nonalcoholic fatty liver disease in 2017," Zhonghua gan zang bing za $z$ hi $=$ Zhonghua ganzangbing $z a z h i=$ Chinese journal of hepatology, vol. 25, no. 9, p. 687, 2017.

[49] I. Trivedi and M. E. Rinella, "NAFLD and cardiovascular disease: Can the real association be determined?" Current Hepatitis Reports, vol. 13, no. 2, pp. 130-141, 2014. 


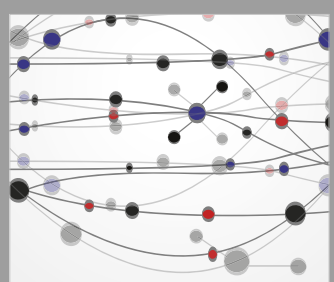

The Scientific World Journal
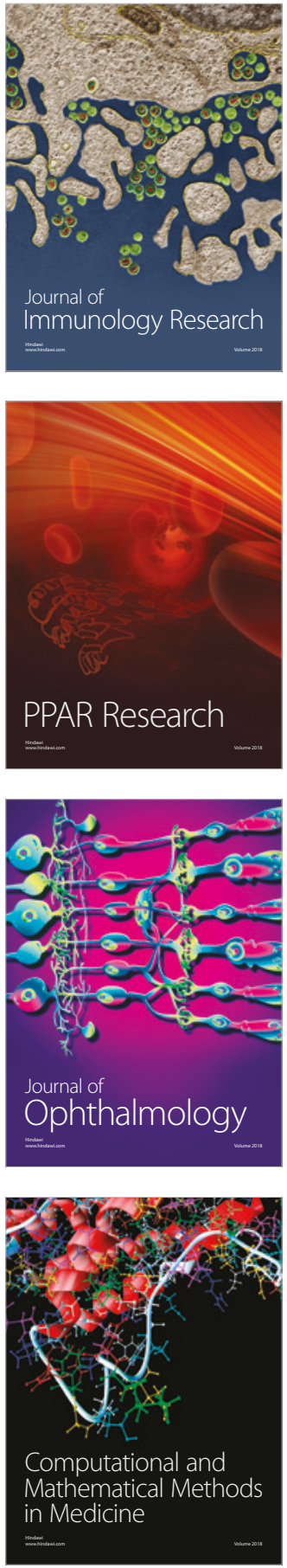

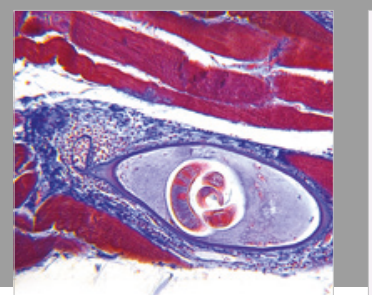

Gastroenterology Research and Practice

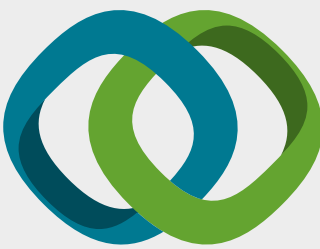

\section{Hindawi}

Submit your manuscripts at

www.hindawi.com
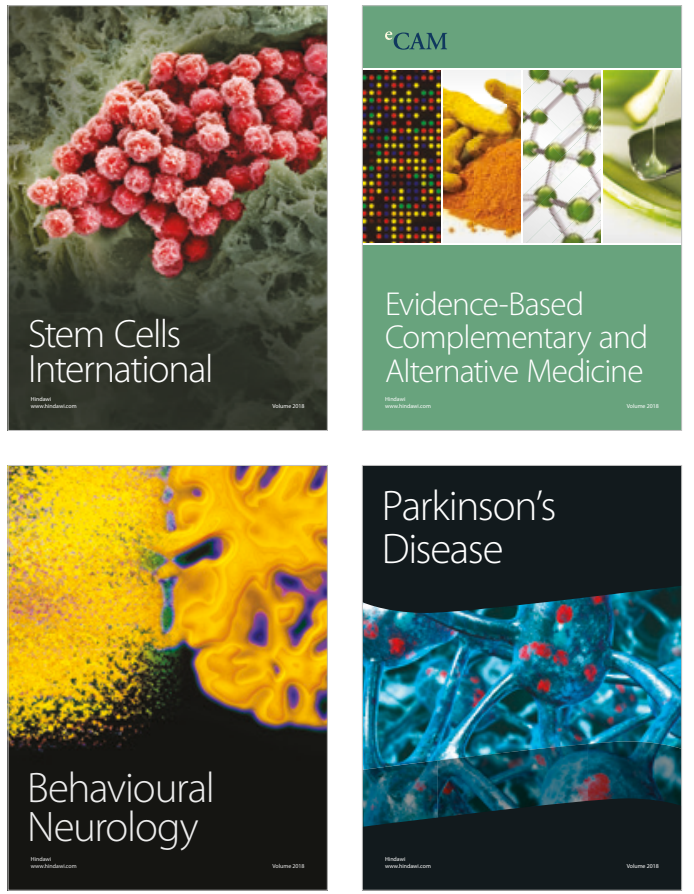

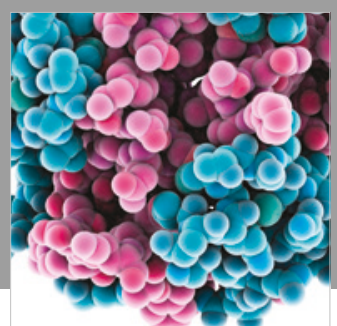

ournal of

Diabetes Research

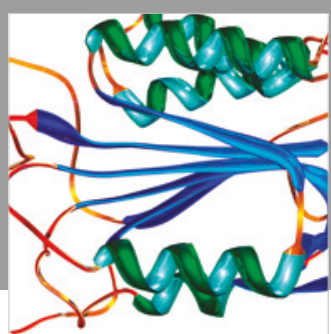

Disease Markers
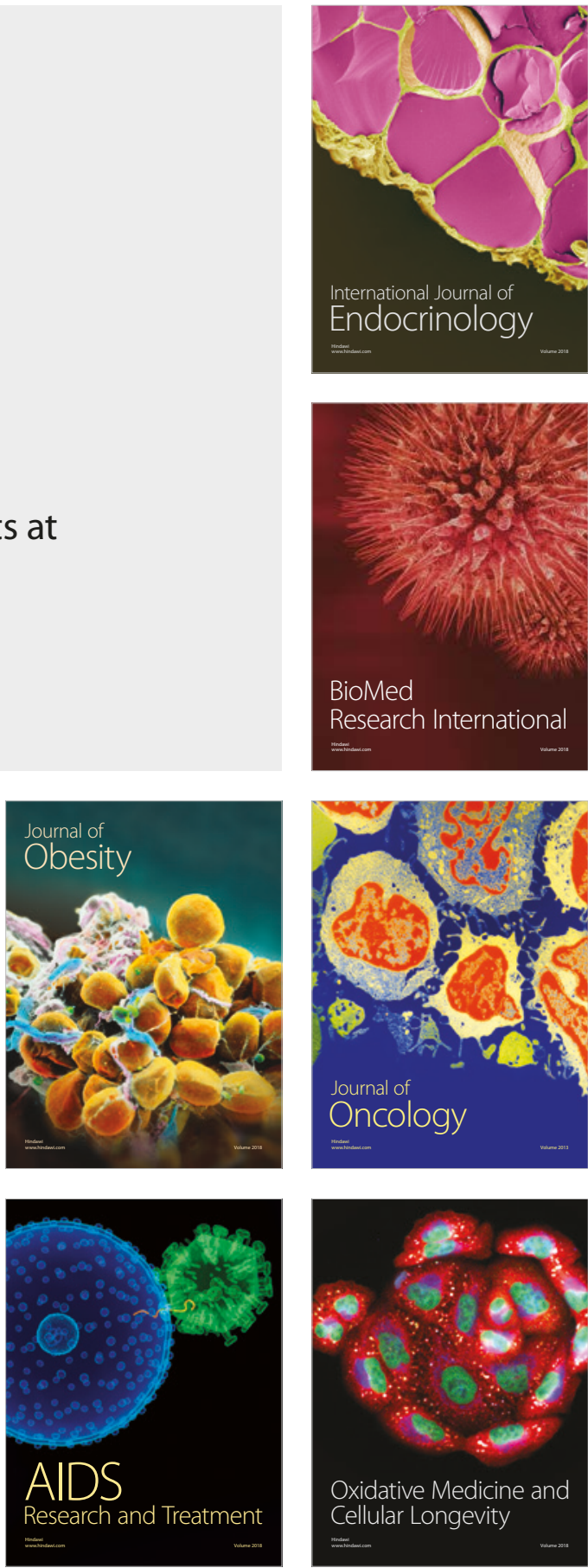